\title{
Impacto de la suplementación con ácidos grasos omega-3 en el embarazo y la reducción del riesgo de parto pretérmino
}

\author{
Salvador Espino y Sosa ${ }^{1 *}$, Enrique Reyes-Muñoz, Gabriela García-Jiménez¹, Abraham Martínez-Ruiz',
} Mario S. Cristo-Aguirre ${ }^{1}$, Ricardo Adame-Pinacho ${ }^{1}$ y Pedro Gutiérrez-Castrellón ${ }^{2}$

${ }^{1}$ Subdirección de Investigación Clínica, Instituto Nacional de Perinatología; ${ }^{2}$ Centro de Investigación Translacional en Ciencias de la Salud, Hospital General Dr. Manuel Gea González; ${ }^{3}$ Sociedad Latinoamericana de Gastroenterologia, Hepatología y Nutrición Pediátrica. Ciudad de México, México

\section{Resumen}

El parto prematuro protagoniza más del $85 \%$ de la morbilidad perinatal, requiere con frecuencia cuidados intensivos y presenta complicaciones que pueden tener consecuencias a lo largo de la vida del individuo. Más de la mitad de los casos de parto pretérmino tienen causas desconocidas y por lo tanto ninguna etiología clara prevenible. Desde la observación en estudios epidemiológicos que demostraron embarazos más largos en poblaciones con alto consumo de aceites marinos se ha tratado de definir por medio de ensayos clínicos aleatorizados el beneficio de suplementos de ácidos grasos poliinsaturados omega-3 (AGPI n-3) prevenir el parto prematuro, así como su valor preventivo. Esta revisión discute la relación entre la suplementación prenatal de n-3 de cadena larga durante el embarazo y la incidencia de parto prematuro.

PALABRAS CLAVE: Embarazo. Ácidos grasos omega 3. Parto pretérmino.

\section{Impact of omega-3 fatty acid during pregnancy and risk reduction for preterm delivery}

\begin{abstract}
Preterm labor accounts for more than $85 \%$ of perinatal morbidity, frequently requiring intensive care and presenting complications that can have consequences throughout the individual's life. More than half of preterm delivery cases have unknown causes and therefore no clear preventable etiology. From observation in epidemiological studies that demonstrated longer pregnancies in populations with high consumption of marine oils, attempts have been made to define the benefit of omega-3 polyunsaturated fatty acids (n-3 PUFA) prevention in premature childbirth through randomized clinical trials, as well as its preventive value. This review discusses the relationship between prenatal supplementation of $n-3$ long chain PUFA during pregnancy and the incidence of preterm delivery.
\end{abstract}

KEY WORDS: Pregnancy. Fatty Acids. Omega-3. Preterm labour.

\section{Introducción}

El parto pretérmino conduce a una alta carga de morbilidad y mortalidad. Cada año nacen en el mundo aproximadamente 15 millones de infantes antes de término ${ }^{1,2}$ y esta es la primera causa de mortalidad y morbilidad neonatal ${ }^{3}$. El interés en los ácidos grasos poliinsaturados omega-3 (AGPI n-3) radica en la cada
Correspondencia:

*Salvador Espino y Sosa

E-mail: salvadorespino@gmail.com
Fecha de recepción: 04-09-2020

Fecha de aceptación: 08-11-2020

DOI: 10.24875/GMM.M20000436
Gac Med Mex. 2020;156(Supl 3): S37-S42

Disponible en PubMed

www.gacetamedicademexico.com 
vez mayor acumulación de reportes que coinciden en su potencial para prevenir enfermedades y promover la salud ${ }^{4}$. Tradicionalmente se han reconocido beneficios potenciales de la suplementación con AG n-3 en el embarazo en dos niveles: reducción de complicaciones propias del embarazo y efecto sobre el neurodesarrollo. El objetivo de la presente revisión es evaluar el efecto de la suplementación de AG n-3 en el embarazo para prevenir el parto prematuro.

\section{Ácidos grasos omega 3 e inflamación}

Los AGPI n-3 comprenden: ácido $\alpha$-linolénico (ALA; 18:3 n-3), ácido estearidónico (SDA; 18:4 n-3), ácido eicosapentaenoico (EPA; 20:5 n-3), ácido docosapentaenoico (DPA; 22:5 n-3) y ácido docosahexaenoico (DHA;22:6 n-3). El efecto protector es complejo, pero guarda fundamento sobre una potencial modulación en la inflamación ${ }^{5}$. En 2004 se reportó una disminución significativa en el riesgo cardiovascular y ciertas formas de cáncer de la población de Groenlandia atribuible a una dieta alta en aceites marinos ${ }^{6}$. A partir de estas publicaciones se iniciaron estudios para evaluar el efecto sobre diversas enfermedades y los últimos años se han vinculado con beneficios en desenlaces tan diversos como microbiota intestinal ${ }^{7}$, epilepsia $^{8}$, retinopatía diabética ${ }^{9}$, depresión ${ }^{10}$, síntomas vasomotores en la menopau$\operatorname{sia}^{11}, \operatorname{cognición}^{12}, \mathrm{asma}^{13}$, etc.

Estas acciones biológicas de los AGPI n-3 están mediadas por enzimas lipídicas producidas por oxigenasas de ácidos grasos tales como ciclooxigenasas (COX), lipoxigenasas (LOX) y monooxigenasas del citocromo P450 (CYP) ${ }^{14}$. El ácido graso omega- 6 araquidónico (AA, 20:4 n-6) se libera de los fosfolípidos de membranas celulares en respuesta a estímulos inflamatorios y es transformado por COX y LOX en eicosanoides: prostaglandinas, leucotrienos y lipoxinas. Los mismos estímulos inflamatorios pueden transformar de igual manera los AGPI n-3 (EPA, DPA y DHA) por la misma vía enzimática compitiendo con la formación de eicosanoides de AA, y proporcionando metabolitos alternativos con actividad menos potente que la de los originales ${ }^{15}$.

La inflamación crónica no controlada puede contribuir en el desarrollo de enfermedades cardiovasculares, autoinmunes, fibrosis y cáncer ${ }^{16}$, por lo que intervenciones en los mecanismos moleculares orientados a controlar la inflamación pueden modificar el curso clínico y pronóstico de estas enfermedades y actuar de modo preventivo.

\section{Inflamación y embarazo}

En el embarazo la inflamación es protagonista de las principales complicaciones del embarazo; preeclampsia y parto pretérmino ${ }^{17-19}$ constituyen verdaderos problemas prioritarios de salud perinatal ${ }^{20-23}$.

Existe evidencia reciente del papel de la inflamación en el parto pretérmino ${ }^{17,18,24}$, dado que el inicio del trabajo de parto fisiológico está íntimamente relacionado con una pérdida del control de la inflamación a nivel local y una estimulación de esta a nivel sistémico ${ }^{25,26}$, es lógico suponer que el parto pretérmino está altamente influido por mecanismos moleculares inflamatorios que pueden ser explicados por un estímulo externo o conocido ${ }^{27}$ y hasta dos terceras partes de los casos ante la ausencia de una etiología identificable ${ }^{1,2}$. Este vínculo estrecho entre inflamación y parto pretérmino es el que ha llevado a diferentes grupos a investigar el efecto de la prevención de la inflamación sobre una disminución en el riesgo de parto pretérmino y el efecto protector de la suplementación de AG n-3 durante el embarazo.

El efecto modulador de la inflamación de los AGPI n-3 se ha asociado a una disminución en el riesgo de complicaciones perinatales, por los hallazgos de estudios epidemiológicos y ensayos clínicos que relacionan el consumo de AGPI n-3 con una mejoría en enfermedades en las que se conoce su relación con inflamación ${ }^{28}$.

Durante el embarazo ocurre una disminución progresiva de las concentraciones séricas de AGPI n-3.

Los AGPI n-3 se transfieren a través de la placenta para el adecuado neurodesarrollo fetal por medio de un transporte activo que facilita concentraciones más altas del omega-3 DHA y el omega- 6 AA, pero un ácido linoleico más bajo (LA, 18:2 n-6) en plasma fetal en relación al plasma materno ${ }^{29}$.

En el estudio clásico de Al, se hizo seguimiento de 110 mujeres mediante tomas de sangre a lo largo del embarazo y al nacimiento muestra de sangre de cordón, así como seis meses después del parto se tomó una muestra de sangre final de la madre. Se determinaron las cantidades absolutas y relativas de los ácidos grasos. Las cantidades totales de ácidos grasos aumentaron significativamente durante el embarazo. Este patrón fue similar para los ácidos grasos individuales y las familias de ácidos grasos. La cantidad relativa de LA no cambió durante el embarazo, mientras que la cantidad relativa de AA disminuyó. A pesar de la movilización materna del DHA, sugerido por un aumento temporal en las concentraciones de DHA 
hasta las 18 semanas de gestación, estas disminuyeron de forma progresiva hasta el fin del embarazo. En general, las concentraciones de ácidos grasos esenciales en circulación materna también disminuyeron constantemente a lo largo de la gestación ${ }^{30}$.

La suplementación durante el embarazo incrementa las concentraciones plasmáticas maternas.

Si bien ocurre un consumo progresivo de AGPI n-3 durante el embarazo por un incremento en las necesidades fetales con un transporte activo transplacentario, la suplementación durante el embarazo se relaciona con un incremento significativo de los niveles plasmáticos.

La suplementación de DHA a razón de $600 \mathrm{mg}$ al día, desde antes de la semana 20 de gestación, aumentan sus concentraciones plasmáticas durante el embarazo y en la sangre del cordón umbilical al nacimiento. El promedio de DHA eritrocitario al final del estudio en el grupo placebo fue de $4.7 \pm 1.3 \%$ y $7.3 \pm 2.2 \%$ en el grupo suplementado ${ }^{31}$. También la dieta materna afecta la acumulación fetal del omega-3 DHA y el omega- $6 \mathrm{AA}^{29}$.

\section{Ácidos grasos poliinsaturados omega-3 y parto pretérmino}

Las primeras asociaciones entre la ingestión de AGPI n-3 y la duración del embarazo datan de la década de 1980, cuando se documentó una mayor duración del embarazo observados en mujeres residentes de Islas Feroe, genéticamente similares a la población de comparación danesa, sugiriendo que los ácidos grasos de los alimentos marinos podrían retrasar el parto espontáneo ${ }^{32,33}$.

\section{Dieta alta en aceites marinos y parto pretérmino}

En estudios epidemiológicos se ha observado una asociación entre una mayor ingestión de pescado y/o suplementos de omega-3 con una disminución en la prevalencia de parto pretérmino. Un estudio nórdico encontró entre mujeres embarazadas que consumían una a dos porciones de pescado por semana una disminución del riesgo de parto pretérmino del $24 \%$ (hazard ratio [HR]: 0.76; intervalo de confianza del 95\% [IC 95\%]: 0.66-0.88) al compararse con mujeres que no consumían pescado y este efecto también se observó al comparar las proporciones de nacimientos lejanos al término por debajo de la semana $32(p=$ $0.05)^{34}$. También se han publicado mecanismos moleculares por los cuales la suplementación/ingesta a AGPI n-3 previene el parto pretérmino, vinculados a la prevención de la activación del inflamasoma uterino ${ }^{35}$.

\section{Efecto de la suplementación en desenlaces perinatales}

Se han publicado múltiples ensayos clínicos que evalúan la administración de aceite de pescado o suplementos de DHA a mujeres embarazadas, con hallazgos heterogéneos.

\section{Estudios a favor de la suplementación con aceites marinos o DHA}

Probablemente el estudio que mejor controla la suplementación y su efecto sobre las concentraciones plasmáticas durante el embarazo para evaluar el efecto de la intervención con los desenlaces es el ensayo de la Dra. Susan Carlston, en el que suplementó a un grupo de mujeres embarazadas desde antes de la semana 20 de gestación con 500 mg diarios de DHA. En comparación con el grupo control, el grupo tratado presentó una menor proporción de nacimiento pretérmino antes de la semana 34 (4.8 vs. $0.6 \% ; p=0.03)$, menos recién nacidos con peso menor a $1,500 \mathrm{~g}$ al nacimiento ( $3.4 \mathrm{vs.} 0 \% ; p=0.03$ ) y menos días de estancia hospitalaria ( $40.8 \pm 44.0$ vs. $8.9 \pm 10.1$ días; $p=0.03$ ). Sin presentar diferencias significativas en la tasa global de nacimiento pretérmino $(8.8 \text { vs. } 7.8 \%)^{31}$.

El metaanálisis Cochrane de la Dra. Maria Makrides incluye 70 estudios con 19,927 participantes ${ }^{36}$. El metaanálisis mostró una reducción del $42 \%$ en el riesgo de nacimiento lejano al término (antes de la semana 34 de gestación) (riesgo relativo [RR]: 0.58; IC 95\%: 0.44-0.77) basado en los hallazgos en nueve ensayos clínicos aleatorizados (ECA) $(n=5,204)$ y una reducción del $11 \%$ en el riesgo de nacimiento pretérmino (RR: 0.89; IC 95\%: 0.81-0.97) en $26 \mathrm{ECA}$ $(n=10,304)$. Se observó un incremento en el riesgo de embarazo postérmino (> 42 semanas; RR: 1.61; IC 95\%: 1.11-2.33) en seis ECA $(n=5,141)$ en los grupos de intervención.

Un metaanálisis de $2016^{37}$ que incluyó más de 10,000 embarazos encontró que el grupo de intervención con aceite de pescado tuvo una reducción del $22 \%$ en el riesgo de nacimiento lejos del término (RR: 0.78; IC 95\%: 0.64-0.95) y una reducción del 
$10 \%$ en el riesgo de parto pretérmino (RR: 0.90; IC 95\%: 0.81-1.00).

Otro metaanálisis de $2016^{38}$ encontró que el riesgo de nacimiento de ePT se redujo en un 58\% (RR: 0.42; IC 95\%: 0.27-0.66) y el nacimiento de PT en un $17 \%$ (RR: 0.83; IC 95\%: 0.70-0.98) por la intervención de aceite de pescado, independientemente de la dosis, el momento de la suplementación o el estado de riesgo del embarazo.

Un metaanálisis de 2012 mostró que la ingestión de aceite de pescado o un suplemento de DHA durante la gestación disminuyó en un $26 \%$ el riesgo de parto lejano al término (RR: 0.74; IC 95\%: 0.58-0.94) sin demostrarse una diferencia significativa en el riesgo de parto pretérmino. (RR: 0.91; IC 95\%: 0.82-1.01). En el grupo de intervención se observó un incremento del peso al nacimiento con significancia estadística, pero una significancia clínica marginal de $42.2 \mathrm{~g}$ (IC 95\%: 14.8-69.7), sin observarse un efecto protector significativo en el riesgo de bajo peso al nacimiento (RR: 0.92; IC 95\%: 0.83-1.02) ${ }^{39}$.

\section{Estudios que no muestran un beneficio de la suplementación}

En 2015 el Dr. Vincenzo Berghella publicó un metaanálisis que comprendió nueve ECA con un total de 3,854 mujeres elegibles. Las mujeres que recibieron omega-3 tuvieron una tasa similar de parto prematuro (antes de las 37 semanas de gestación) en comparación con las mujeres del grupo de control (7.7 vs. 9.1\%; RR: 0.90; IC 95\%: 0.72-1.11). No se observaron diferencias en el peso al nacimiento, ingreso a la unidad de cuidados intensivos neonatales, enterocolitis necrotizante, sepsis o muerte neonatal. No hubo diferencias significativas en los análisis de subgrupos, excepto por la tasa de muerte perinatal, que fue más baja (0.3\% en comparación con 1.2\%; RR: 0.27; IC 95\%: $0.09-0.80$ ) en las mujeres que recibieron omega-3 antes de las 21 semanas de gestación y en ensayos con bajo riesgo de sesgo ( $0.3 \%$ en comparación con 1.0\%; RR: 0.28; IC 95\%: 0.09-0.89) en comparación con las mujeres en el grupo control. Sin embargo, en ningún ensayo clínico aleatorizado la muerte perinatal fue el resultado primario ${ }^{29}$.

En el año 2019, la Dra. María Makrides, autora de la revisión sistemática Cochrane de 2018, publicó un ECA, un total de 5,544 embarazos en 5,517 mujeres (algunas de ellas se embarazaron dos veces) fueron asignados al azar a recibir aceite de pescado conteniendo $900 \mathrm{mg}$ de AGPI n-3 o aceite vegetal en seis centros en Australia; se incluyeron 5,486 embarazos en el análisis primario. El grupo de intervención presentó 61 casos de parto pretérmino de los 2,734 embarazos incluidos (2.2\%) y el grupo control reportó 55 de 2,752 embarazos (2.0\%); la diferencia entre grupos no fue significativa (RR: 1.13 ; IC 95\%: 0.791.63; $p=0.50$ ). Tampoco se encontraron diferencias significativas en la incidencia de intervenciones por embarazo postérmino (> 41 semanas de gestación), otros eventos adversos del embarazo o en resultados neonatales, con la salvedad del hallazgo de una mayor proporción de recién nacidos grandes para la edad gestacional en el grupo intervenido (RR ajustado: 1.30; IC 95\%: 1.02-1.65). Se reportó una mayor incidencia de molestias digestivas en el grupo intervenido, sin consecuencias de graves ${ }^{40}$.

\section{¿La eficacia de la intervención depende de la población estudiada?}

La controversia de los resultados entre estudios de investigación ha sido abordados y explicados por el grupo del Dr. Timothy $\mathrm{H}$. Ciesielski, utilizando datos poblacionales de 184 países, analizando la relación entre las tasas nacionales de parto pretérmino $(<37$ semanas de gestación) y las normas de ingesta de AGPI n-3 para el año 2010. Los autores estimaron los niveles de AG n-3 utilizando un estimador que incluye la ingesta de omega-3 a base de mariscos (EPA y DHA) y la ingesta de omega-3 a base de plantas (ALA), considerando que aproximadamente el $20 \%$ de estos últimos se convierte en EPA/DHA in vivo. El análisis demostró que las tasas de parto pretérmino entre países disminuyen linealmente al aumentar los niveles de omega-3 hasta aproximadamente $600 \mathrm{mg} / \mathrm{día}$. El análisis de regresión lineal ajustado por ingresos entre los países en este rango de exposición indicó que el número de partos antes de la semana 37 por 100 nacidos vivos disminuyó en 1.5 (IC 95\%: 2.8-0.3) por cada aumento de una desviación estándar en la ingesta de omega-3 $(383 \mathrm{mg} /$ día $)^{41}$. Estos hallazgos sugieren que la eficacia de la intervención podría modificarse por la cantidad diaria de omega-3 que una población ingiere, beneficiando únicamente aquellas que cuentan con una menor de $600 \mathrm{mg}$ diarios.

Una revisión sistemática de 151,880 embarazos en Europa mostró que las mujeres gestantes que comieron pescado en más de una ocasión a la semana redujeron la probabilidad de nacimiento pretérmino al 
ser comparadas con aquellas que solo lo hacían de forma ocasional ${ }^{42}$, y es que poblaciones con una concentración baja de AGPI n-3 (EPA y DHA) durante el embarazo (menor al $2 \%$ ) incrementan de forma significativa el riesgo de parto pretérmino. Un estudio de casos y controles anidado encontró que las mujeres en el quintil más bajo (EPA + DHA < 1.6\%) tuvieron 10.27 veces mayor riesgo (IC 95\%: 6.80-15.79; $\mathrm{p}<0.0001)$ y las mujeres en el segundo quintil más bajo tuvieron 2.86 veces mayor riesgo (IC 95\%: 1.794.59; $p<0.0001$ ), en comparación con las mujeres en los tres quintiles más altos agregados (EPA + DHA $\geq 1.8 \%)^{32}$.

Estos hallazgos motivaron a identificar un biomarcador de AGPI omega-3 capaz de detectar qué mujeres con embarazos únicos tienen más probabilidades de beneficiarse de la suplementación con omega-3 para reducir su riesgo de parto prematuro temprano. Un análisis exploratorio de un ensayo controlado aleatorizado realizado en seis hospitales australianos identificó AGPI n-3 en sangre capilar materna en la semana 14 de gestación, y encontraron que concentraciones bajas de AGPI omega-3 al comienzo del embarazo constituyen un importante factor de riesgo de parto prematuro temprano. En las mujeres con concentraciones relativas $\leq 4.1 \%$ del total de ácidos grasos, la suplementación con omega-3 redujo sustancialmente el riesgo de parto prematuro temprano en comparación con el control (0.73 vs. 3.16\%; RR: 0.23; IC 95\%: 0.07-0.79). Por el contrario, las mujeres con un estado omega-3 total más alto al comienzo del embarazo tenían un menor riesgo de parto prematuro temprano. Complementar a las mujeres con un estado basal superior al 4.9\% aumentó el parto prematuro temprano (2.20 vs. 0.97\%; RR: 2.27; IC 95\%: 1.13$4.58)^{43}$. Las mujeres con embarazos únicos y bajo estado total de AGPI omega-3 al inicio del embarazo tienen un mayor riesgo de parto prematuro temprano y es más probable que se beneficien de los suplementos de omega-3 para reducir este riesgo. Las mujeres con un estado omega- 3 total más alto tienen un riesgo menor y la suplementación adicional con omega-3 puede aumentar su riesgo.

Esto establece que concentraciones plasmáticas bajas de EPA y DHA durante el embarazo constituyen un factor de riesgo importante para desarrollar parto prematuro. Desde esta perspectiva, la suplementación con AG n-3 tendría un efecto protector en poblaciones con bajo consumo. En Ciudad de México, en una cohorte de mujeres embarazadas se cuantificó la ingestión de diferentes AGPI en los tres trimestres del embarazo utilizando el cuestionario estándar de frecuencia de alimentos. En el primero y segundo trimestres, el percentil $50 \%$ de consumo se reportó en 20 mg/día de EPA y $40 \mathrm{mg} /$ día para DHA, en el tercer trimestre el percentil $50 \%$ de consumo se reportó en $10 \mathrm{mg} /$ día de EPA y $40 \mathrm{mg} /$ día para $\mathrm{DHA}^{44}$. Estos hallazgos categorizan a la población de la Ciudad de México como de baja ingesta de AG n-3 y bajo la lógica del estudio de Ciesielski se vería beneficiada de una intervención de suplementación.

\section{Financiamiento}

La presente investigación no ha recibido ninguna beca específica de agencias de los sectores público, comercial, o sin ánimo de lucro.

\section{Conflicto de intereses}

El autor principal Salvador Espino y Sosa es asesor científico de Healthcare Nutrition.

\section{Responsabilidades éticas}

Protección de personas y animales. Los autores declaran que para esta investigación no se han realizado experimentos en seres humanos ni en animales.

Confidencialidad de los datos. Los autores declaran que han seguido los protocolos de su centro de trabajo sobre la publicación de datos de pacientes.

Derecho a la privacidad y consentimiento informado. Los autores declaran que en este artículo no aparecen datos de pacientes

\section{Bibliografía}

1. Gilman-Sachs A, Dambaeva S, Salazar Garcia MD, Hussein Y, KwakKim J, Beaman K. Inflammation induced preterm labor and birth. J Reprod Immunol. 2018;129:53-8.

2. Vogel JP, Chawanpaiboon S, Moller AB, Watananirun K, Bonet M, Lumbiganon $\mathrm{P}$. The global epidemiology of preterm birth. Best Pract Res Clin Obstet Gynaecol. 2018;52:3-12.

3. Harrison MS, Goldenberg RL. Global burden of prematurity. Semin Fetal Neonatal Med. 2016;21(2):74-9.

4. Shahidi $F$, Ambigaipalan P. Omega-3 polyunsaturated fatty acids and their health benefits. Annu Rev Food Sci Technol. 2018;9:345-81.

5. Calder PC. Omega-3 fatty acids and inflammatory processes: from molecules to man. Biochem Soc Trans. 2017;45(5):1105-15.

6. Shahidi F, Miraliakbari H. Omega-3 (n-3) fatty acids in health and disease: Part 1-Cardiovascular disease and cancer. J Med Food. Winter 2004;7(4):387-401.

7. Costantini L, Molinari R, Farinon B, Merendino N. Impact of omega-3 fatty acids on the gut microbiota. Int J Mol Sci. 2017;18(12).

8. Tejada S, Martorell M, Capó X, Tur JA, Pons A, Sureda A. Omega-3 fatty acids in the management of epilepsy. Curr Top Med Chem. 2016;16(17):1897-905

9. Behl T, Kotwani A. Omega-3 fatty acids in prevention of diabetic retinopathy. J Pharm Pharmacol. 2017;69(8):946-54.

10. Grosso G, Galvano F, Marventano S, Malaguarnera M, Bucolo C, Drago $F$, et al. Omega-3 fatty acids and depression: scientific evidence and biological mechanisms. Oxid Med Cell Longev. 2014;2014:313570. 
11. Mohammady M, Janani L, Jahanfar S, Mousavi MS. Effect of omega-3 supplements on vasomotor symptoms in menopausal women: A systematic review and meta-analysis. Eur J Obstet Gynecol Reprod Biol. 2018;228:295-302.

12. Rangel-Huerta OD, Gil A. Effect of omega-3 fatty acids on cognition: an updated systematic review of randomized clinical trials. Nutr Rev. 2018;76(1):1-20.

13. Miyata $\mathrm{J}$, Arita M. Role of omega- 3 fatty acids and their metabolites in asthma and allergic diseases. Allergol Int. 2015;64(1):27-34

14. Isobe $Y$, Arita M. Identification of novel omega-3 fatty acid-derived bioactive metabolites based on a targeted lipidomics approach. J Clin Biochem Nutr. 2014:55(2):79-84.

15. Naoe S, Tsugawa H, Takahashi M, Ikeda K, Arita M. Characterization of lipid profiles after dietary intake of polyunsaturated fatty acids using integrated untargeted and targeted lipidomics. Metabolites. 2019:9:241.

16. Gupta SC, Prasad S, Aggarwal BB. Anti-inflammatory nutraceuticals and chronic diseases. Springer; 2016.

17. Akkaya Fırat A, Alıcı Davutoğlu E, Özel A, Güngör ZB, Madazlı R, Ulakoğlu Zengin E. Hypoxia-inducible factor- $1 \alpha$, hepcidin and interleukin-6 levels in pregnancies with preterm labour. J Obstet Gynaecol. 2020;40(6):813-9.

18. Robertson SA, Hutchinson MR, Rice KC, Chin P-Y, Moldenhauer LM, Stark MJ, et al. Targeting toll-like receptor-4 to tackle preterm birth and fetal inflammatory injury. Clin Transl Immunology. 2020;9(4):e1121.

19. Bhatti G, Romero R, Rice GE, Fitzgerald W, Pacora P, Gomez-Lopez N, et al. Compartmentalized profiling of amniotic fluid cytokines in women with preterm labor. PLoS One. 2020;15(1):e0227881.

20. Mihu D, Razvan C, Malutan A, Mihaela C. Evaluation of maternal systemic inflammatory response in preeclampsia. Taiwan J Obstet Gynecol. 2015;54(2):160-6

21. Wang Y, Dong Q, Gu Y, Groome LJ. Up-regulation of miR-203 expression induces endothelial inflammatory response: Potential role in preeclampsia. Am J Reprod Immunol. 2016;76(6):482-90.

22. Vaughan JE, Walsh SW. Activation of NF-KB in placentas of women with preeclampsia. Hypertens Pregnancy. 2012;31(2):243-51

23. Cakmak HA Dincgez Cakmak B, Abide Yayla C, Inci Coskun E, Erturk M, Keles I. Assessment of relationships between novel inflammatory markers and presence and severity of preeclampsia: Epicardial fat thickness, pentraxin-3, and neutrophil-to-lymphocyte ratio. Hypertens Pregnancy. 2017;36(3):233-9.

24. Tedesco RP, Galvão RB, Guida JP, Passini-Júnior R, Lajos GJ, Nomura ML, et al. The role of maternal infection in preterm birth: evidence from the Brazilian Multicentre Study on Preterm Birth (EMIP). Clinics. 2020:75:e1508.

25. Nguyen-Ngo C, Lappas M. Mechanisms of normal labour. Curr Opin Physiol. 2020;13:27-32.

26. Sivarajasingam SP, Imami N, Johnson MR. Myometrial cytokines and their role in the onset of labour. J Endocrinol. 2016:231(3):R101-19.

27. Amabebe E, Reynolds S, He X, Wood R, Stern V, Anumba DOC. Infection/inflammation-associated preterm delivery within 14 days of presentation with symptoms of preterm labour: A multivariate predictive model. PLoS One. 2019;14(9):e0222455.

28. Isobe $\mathrm{Y}$, Arita M. Omega-3 fatty acid metabolism and regulation of inflammation. En: Yokomizo T, Murakami M, editores. Bioactive Lipid Mediators. Tokyo: Springer; 2015. pp. 155-162.
29. Saccone G, Berghella V. Omega-3 supplementation to prevent recurrent preterm birth: a systematic review and metaanalysis of randomized controlled trials. Am J Obstet Gynecol. 2015;213(2):135-40.

30. Al MD, van Houwelingen AC, Kester AD, Hasaart $T H$, de Jong $A E$, Hornstra G. Maternal essential fatty acid patterns during normal pregnancy and their relationship to the neonatal essential fatty acid status. Br J Nutr. 1995;74(1):55-68.

31. Carlson SE, Colombo J, Gajewski BJ, Gustafson KM, Mundy D, Yeast J, et al. DHA supplementation and pregnancy outcomes. Am J Clin Nutr. 2013;97(4):808-15.

32. Olsen SF, Halldorsson TI, Thorne-Lyman AL, Strøm M, Gørtz S, Granstrøm C et al. Plasma concentrations of long chain n-3 fatty acids in early and mid-pregnancy and risk of early preterm birth. EBioMedicine. 2018;35:325-33.

33. Olsen SF, Hansen HS, Secher NJ, Jensen B, Sandström B. Gestation length and birth weight in relation to intake of marine $n-3$ fatty acids. $\mathrm{Br}$ J Nutr. 1995;73(3):397-404.

34. Brantsæter AL, Englund-Ögge L, Haugen M, Birgisdottir BE, Knutsen HK, Sengpiel V, et al. Maternal intake of seafood and supplementary long chain n-3 poly-unsaturated fatty acids and preterm delivery. BMC Pregnancy Childbirth. 2017; 17(1):41.

35. Chen C-Y, Chen C-Y, Liu C-C, Chen C-P. Omega-3 polyunsaturated fatty acids reduce preterm labor by inhibiting trophoblast cathepsin $\mathrm{S}$ and inflammasome activation. Clin Sci . 2018;132(20):2221-39.

36. Middleton P, Gomersall JC, Gould JF, Shepherd E, Olsen SF, Makrides M. Omega-3 fatty acid addition during pregnancy. Cochrane Database Syst Rev. 2018;11:CD003402.

37. Chen B, Ji X, Zhang L, Hou Z, Li C, Tong Y. Fish oil supplementation improves pregnancy outcomes and size of the newborn: a meta-analysis of 21 randomized controlled trials. J Matern Fetal Neonatal Med. 2016;29(12):2017-27.

38. Kar S, Wong M, Rogozinska E, Thangaratinam S. Effects of omega-3 fatty acids in prevention of early preterm delivery: a systematic review and meta-analysis of randomized studies. Eur J Obstet Gynecol Reprod Biol. 2016:198:40-6.

39. Imhoff-Kunsch B, Briggs V, Goldenberg T, Ramakrishnan U. Effect of n-3 long-chain polyunsaturated fatty acid intake during pregnancy on maternal, infant, and child health outcomes: a systematic review. Paediatr Perinat Epidemiol. 2012;26(Suppl 1):91-107.

40. Makrides M, Best K, Yelland L, McPhee A, Zhou S, Quinlivan J, et al. A randomized trial of prenatal $n-3$ fatty acid supplementation and preterm delivery. N Engl J Med. 2019;381(11):1035-45.

41. Ciesielski TH, Bartlett J, Williams SM. Omega-3 polyunsaturated fatty acid intake norms and preterm birth rate: a cross-sectional analysis of 184 countries. BMJ Open. 2019;9(4):e027249.

42. Leventakou V, Roumeliotaki T, Martinez D, Barros H, Brantsaeter A-L, Casas $M$, et al. Fish intake during pregnancy, fetal growth, and gestational length in 19 European birth cohort studies. Am J Clin Nutr. 2014:99(3):506-16.

43. Simmonds LA, Sullivan TR, Skubisz M, Middleton PF, Best KP, Yelland LN, et al. Omega-3 fatty acid supplementation in pregnancy-baseline omega-3 status and early preterm birth: exploratory analysis of a randomised controlled trial. BJOG. 2020;127(8):975-81.

44. Al-Hinai M, Baylin A, Tellez-Rojo MM, Cantoral A, Ettinger $A$, Solano-González M, et al. Maternal intake of omega-3 and omega-6 polyunsaturated fatty acids during mid-pregnancy is inversely associated with linear growth. J Dev Orig Health Dis. 2018;9(4):432-41. 